\title{
Article \\ Application of Tucker Decomposition in Temperature Distribution Reconstruction
}

\author{
Zhaoyu Liu ${ }^{1}$, Shi Liu ${ }^{2, *}$, Minxin Chen ${ }^{3}$, Yaofang Zhang ${ }^{1}$ and Pengbo Yao ${ }^{1}$ \\ 1 School of Energy, Power and Mechanical Engineering, North China Electric Power University, \\ Beijing 102206, China; liuzhaoyu@ncepu.edu.cn (Z.L.); zhangyaofang@ncepu.edu.cn (Y.Z.); \\ yaopengbo718@ncepu.edu.cn (P.Y.) \\ 2 School of Control and Computer Engineering, North China Electric Power University, Beijing 102206, China \\ 3 Development Research Centre of State Power Investment Group Co., Ltd., Beijing 102206, China; \\ chenminxin@spic.com.cn \\ * Correspondence: liushi@ncepu.edu.cn; Tel.: +86-010-6177-2859
}

check for updates

Citation: Liu, Z.; Liu, S.; Chen, M.; Zhang, Y.; Yao, P. Application of Tucker Decomposition in Temperature Distribution Reconstruction. Appl. Sci. 2022, 12, 2749. https://doi.org/10.3390/ app12052749

Academic Editor:

Georgios Karavalakis

Received: 25 January 2022

Accepted: 4 March 2022

Published: 7 March 2022

Publisher's Note: MDPI stays neutral with regard to jurisdictional claims in published maps and institutional affiliations.

Copyright: (C) 2022 by the authors. Licensee MDPI, Basel, Switzerland. This article is an open access article distributed under the terms and conditions of the Creative Commons Attribution (CC BY) license (https:// creativecommons.org/licenses/by/ $4.0 /)$.

\begin{abstract}
Constrained by cost, measuring conditions and excessive calculation, it is difficult to reconstruct a 3D real-time temperature field. For the purpose of solving these problems, a threedimensional temperature distribution reconstruction algorithm based on Tucker decomposition algorithm is proposed. The Tucker decomposition algorithm is used to reduce the dimension of the measured data, and the processed core tensor is used for the temperature field reconstruction of sparse data. Theoretical analysis and simulations show that the proposed method is feasible; the overall optimization is realized by selecting the appropriate core tensor dimensions; and the reconstruction error is less than 3\%. Results indicate that the proposed method can yield a reliable reconstruction solution and can be applied to real-time applications.
\end{abstract}

Keywords: Tucker decomposition; three-dimensional temperature distribution; core tensor; dimensionality reduction

\section{Introduction}

Accurate reliable measurements of 3D flame temperature distribution are highly desirable to achieve an in-depth understanding of combustion and pollutant formation processes including flame flashback prediction, thermoacoustic instability control and combustion emission reduction. YU Guan et al. experimentally studied and verified that the periodic acoustic forcing effect [1] and strategic use of transient forcing, hysteresis and mode switching can reduce the thermoacoustic amplitude of the self-excited combustion system [2]. On this basis, the application scope of open-loop control was explored [3]. C. L. Myung et al. reviewed the particulate emission characteristics of internal combustion engine exhaust [4] and the spark ignition and diesel engine combustion process [5] and compared the effects of different vehicle certification modes on particulate emission characteristics of light vehicles through experiments [6]. C. Heeger et al. explored the mechanism of flame flashback in annular slots of burners and put forward a new hypothesis of tempering mechanism [7]. A. Nauert et al. used planar laser diagnostics at high repetition rates to quantitatively study the flame during flashback [8]. F. Seffrin et al. proposed a layered burner, verified the numerical simulation model through experiments and analyzed the flow field of the burner. The experimental apparatus and results are discussed [9]. Due to the thermal and dynamic nature of the flame, an effective means for the spatial and temporal measurement of the flame temperature remains a challenge for combustion and measurement researchers.

Significant efforts have been made in past decades to measure the flame temperature distribution. Various contact and non-contact methods have been proposed and developed. There are numerous methods for measuring temperature. The contact temperature measurement methods mainly refer to the thermocouples, widely used in the combustion 
process $[10,11]$. It is convenient and efficient, but it can only be used for single-point measurements. It is impossible to install enough equipment for detailed information. Non-contact measurement methods, such as acoustic measurement [12], laser diagnostic measurement [13] and radiation imaging methods [14], can be used to reconstruct the temperature field. These techniques have the advantages of a wide measurement range, fast dynamic response and little impact on the flame $[15,16]$. However, these methods can generally measure the parameters on the line or surface, and many problems remain that may limit their application; for example, noise, vibration, and ash in the industrial environment may have a great impact on their accuracy. Furthermore, sensors involved in these techniques are difficult to install, and the measurement systems are complicated and expensive. All of these factors increase the difficulty of applying these devices. Numerical methods, such as computational fluid dynamics (CFD), can also be used to obtain accurate and detailed temperature information. However, numerical computations always involve a large amount of data; they are exceptionally time-consuming and not suitable for real-time applications. Therefore, it is significant to propose and develop an effective method to use these data.

By extracting eigenvector by learning from experience dataset, principal component analysis (PCA) — also known as Karhunen-Loève (KL) expansion or proper orthogonal decomposition (POD) — can analyze and utilize the most essential characteristics of these data.

As the PCA is convenient and efficient, it is used in many different domains, including signal analysis [17], data compression [18], image processing [19], chemical reaction analysis [20] and control systems [21]. In recent years, it has also been well used in 2D temperature distribution and wind field analysis and reconstruction. Chen et al. [22] combines measurement and numerical algorithms based on PCA to obtain temperature distribution in real time by using feature vectors and measurement data. Sun et al. [23] used PCA for real-time wind field reconstruction for large areas and improving the efficiency of sensor applications.

In order to develop a 3D temperature field reconstruction method that can be used in real applications, inspired by the PCA, a three-dimensional temperature distribution reconstruction algorithm based on Tucker decomposition algorithm is proposed.

Tucker decomposition was first proposed by Tucker in 1963 [24]. Since then, Tucker decomposition has been applied in many different fields, including data compression [25], image processing [26], signal analysis [27] and so on. Zhao et al. [28] used it for infrared small-target detection, and Qin et al. [29] used it in wind velocity distribution reconstruction. The method has also been used in hyperspectral change detection [30].

In this study, a method based on Tucker decomposition algorithm is proposed for the rapid and reliable reconstruction of the 3D temperature field using sparse measurements, and it was verified by simulation data and experimental data, which provides a new solution for temperature distribution measurement.

\section{Fundamental Measurement Principles}

\subsection{Tucker Decomposition Algorithm Principle}

By Tucker decomposition, the N-order high-dimensional tensor can be transformed into an n-order low-dimensional core tensor and $\mathrm{n}$ decomposition factor matrices $(N>n)$. The Tucker decomposition of N-order tensors $\chi \in \complement^{I \times J \times K}$ can be written as [31]

$$
\chi=f\left(U^{(1)}, U^{(2)}, \ldots, U^{(N)}\right)+\varepsilon
$$

where $U^{(n)}, n=1, \ldots, N$ is the decomposition factor, and $\varepsilon$ is $\mathrm{N}$-order error tensor.

Therefore, the factor matrix can be obtained by the following optimization problems:

$$
\left(\hat{U}^{(1)}, \ldots, \hat{U}^{(N)}\right)=\arg \min _{U^{(1)}, \ldots, U^{(N)}}\left\|\chi-f\left(U^{(1)}, \ldots, U^{(N)}\right)\right\|_{2^{\prime}}^{2}
$$


Considering that the Tucker decomposition of the N-order tensor is a higher-order singular value decomposition (HOSVD),

$$
\min _{U^{(1)}, \ldots, U^{(N)}, G_{(n)}}\left\|X_{(n)}-U^{(n)} G_{(n)} U_{\otimes}^{(n)}\right\|_{2^{\prime}}^{2}
$$

In higher-order SVD, the factor matrix $U^{(n)} \in \complement^{I_{n} \times J_{n}}$ satisfies the semi orthogonal condition $U^{(n) T} U^{(n)}=I_{J}$ :

$$
U_{\otimes}^{(n)} U_{\otimes}^{(n) T}=I_{J_{1}, \ldots, J_{n-1}, J_{n+1}, \ldots, J_{N},}
$$

Then, to solve equation $X_{(n)} \approx U^{(n)} G_{(n)} U_{\otimes}^{(n)}$, make SVD $X_{(n)}=U^{(n)} S^{(n)} V^{(n) T}$ :

$$
U^{(n)} S^{(n)} V^{(n) T}=U^{(n)} G_{(n)} U_{\otimes}^{(n)},
$$

Then, both sides multiply $U^{(n) T}$ left and $U_{\otimes}^{(n) T}$ right, respectively, and the following can be obtained from Equation (5):

$$
G_{(n)}=S^{(n)} V^{(n) T} U_{\otimes}^{(n) T},
$$

Therefore, the mathematical model of the n-order tensor $\chi \in \complement^{I_{1} \times I_{2} \times I_{3} \times \ldots I_{N}}$ decomposition process is expressed as follows:

$$
\chi=\zeta \times_{1} U^{(1)} \times_{2} U^{(2)} \ldots \times_{N} U^{(N)},
$$

where $\zeta$-tensor $\chi$ Tucker decomposition core tensor, $\zeta \in \complement^{J_{1}} \times \mathrm{J}_{2} \times \mathrm{J}_{3} \times \ldots \times \mathrm{J}_{\mathrm{N}}, J_{N}<I_{N} ; \times{ }_{N}-$ tensor $\chi N$-mode product and $\boldsymbol{U}^{(N)}$ — tensor $\chi$ decomposition factor matrix in the N-module direction, $\boldsymbol{U}^{(N)} \in \complement^{I_{n} \times J_{n}}$.

\subsection{Reconstruction Algorithm Based on Tucker Decomposition}

In the reconstruction of three-dimensional temperature distribution, the a priori dataset is composed of $n_{1}$ three-dimensional temperature field obtained by simulation calculation or measurement. In order to facilitate the subsequent calculation and analysis, the prior dataset is recorded as a fourth-order tensor $T R=\left\{T_{1}, T_{2}, T_{3}, \ldots, T_{i}, \ldots, T_{n 1}\right\}, T_{i} \in$ $\complement^{n_{2} \times n_{3} \times n_{4}}, \boldsymbol{T R} \in \mathcal{C}^{n_{1} \times n_{2} \times n_{3} \times n_{4}}$. The prior dataset is decomposed into the form of core tensor and decomposition factor mode product through Tucker decomposition, as follows:

$$
T R=\left\{\begin{array}{c}
T_{1} \\
T_{2} \\
\vdots \\
T_{\mathrm{i}} \\
\vdots \\
T_{n_{1}}
\end{array}\right\}=C_{t} \times{ }_{1} F_{t(1)} \times{ }_{2} F_{t(2)} \times{ }_{3} F_{t(3)} \times{ }_{4} F_{t(4)}
$$

where $C_{t}$-the core tensor of prior dataset tensor TR Tucker decomposition, $\boldsymbol{C}_{\boldsymbol{t}} \in \mathrm{C}^{m_{1} \times m_{2} \times m_{3} \times m_{4}}$ and $\boldsymbol{F}_{\boldsymbol{t}(\boldsymbol{i})}$-decomposition factor matrix in the $\boldsymbol{i}$-module direction of tensor $\boldsymbol{T R}, \boldsymbol{F}_{\boldsymbol{t}(\boldsymbol{i})} \in \complement^{n_{i} \times m_{i}}$.

$$
\mathrm{C} \times{ }_{1} P \times_{2} Q=C \times{ }_{2} Q \times{ }_{1} P
$$

where $\boldsymbol{P}$ - the decomposition factor matrix in the 1-mode direction and $\mathrm{Q}$ - the decomposition factor matrix in the 2-mode direction. 
According to Formula (10), Formula (9) is transformed into the following form:

$$
T R=\left\{\begin{array}{c}
T_{1} \\
T_{2} \\
\vdots \\
T_{i} \\
\vdots \\
T_{n_{1}}
\end{array}\right\}=C_{t} \times{ }_{1} F_{t(1)} \times{ }_{2} F_{t(2)} \times{ }_{3} F_{t(3)} \times{ }_{4} F_{t(4)} \times{ }_{1}\left\{\begin{array}{c}
f_{11} \\
f_{12} \\
\vdots \\
f_{1 i} \\
\vdots \\
f_{1 n_{1}}
\end{array}\right\},
$$

where $f_{1 i}$-the $i$ th factor vector in the decomposition factor matrix in the direction of 1 modulus and $f_{1 i} \in \complement^{1 \times m_{1}}$.

It can be seen from Formula (11):

$$
T_{i}=A \times{ }_{1} f_{1 i}
$$

where $A$-reconstruction coefficient tensor, $A=C \times_{2} F_{(2)} \times{ }_{3} F_{(3)} \times{ }_{4} F_{(4)}$ and $\boldsymbol{A} \in \mathrm{C}^{m_{1} \times n_{2} \times n_{3} \times n_{4}}$.

In the process of solving, for the three-dimensional temperature distribution to be reconstructed under the same combustion environment, the Equation (13) should also be satisfied:

$$
T_{x}=A \times{ }_{1} f_{1 x^{\prime}}
$$

where $f_{1 x}$-the three-dimensional temperature distribution to be solved to reconstruct the 1-mode decomposition factor vector.

According to the definition of model product, the Formula (13) is transformed into the form of vector and matrix multiplication:

$$
t_{x}=\left(f_{1 x} \times A_{1 \text { unfold }}\right)^{T},
$$

where $\boldsymbol{t}_{x}$-first-order representation of three-dimensional temperature distribution; $A_{1 \text { unfold }}$ the expansion of the coefficient matrix A along the direction of 1 modulus and $A_{1 \text { unfold }} \in$ $\complement^{\left(n_{2} \times n_{3} \times n_{4}\right) \times m_{1}}$

In the temperature measurement process, when the $\mathrm{k}$ measuring point positions are determined, the corresponding measurement matrix can be constructed, and the temperature measurement process can be expressed as a mathematical model:

$$
t_{M}=M t_{x}
$$

where $t_{M}$-a vector of small number of temperature measurements, $t_{M} \in R^{k \times \mathbf{1}} ; M$-the measurement matrix, which characterizes the location of the measurement points and $M \in R^{k \times n}$.

Combining Formula (14) and Formula (15), the relationship between the measured temperature and the coefficient vector to be solved can be established:

$$
M A_{1 \text { unfold }} f_{1 x}^{T}=t_{M}
$$

In Formula (16), $t_{M}$ is obtained by measurement, $M$ is a known condition after the position of the measuring point is fixed, and $A_{1 \text { unfold }}$ can be calculated by Tucker decomposition of the prior data set. Set the objective function of minimizing reconstruction error based on the measured data:

$$
J\left(f_{1 x}^{T}\right)=\arg \min \left\|t_{M}-M A_{1 \text { unfold }}^{T} f_{1 x}^{T}\right\|^{2},
$$

where $J\left(f_{1 x}^{T}\right)$-the objective function. 
Through Formula (17), the temperature distribution reconstruction is transformed into a problem of obtaining the optimal solution of the reconstruction coefficient vector:

$$
B f_{1 x}^{T}=k,
$$

where $B=\left(M A_{1 \text { unfold }}^{T}\right)^{T}\left(M A_{1 \text { unfold }}^{T}\right) ; k=\left(M A_{1 \text { unfold }}^{T}\right)^{T} t_{M}$.

Solve the Formula (18), and then calculate the Formula (14) to obtain the threedimensional reconstructed temperature distribution. The specific process of temperature field reconstruction by Tucker decomposition is shown in Figure 1 below.

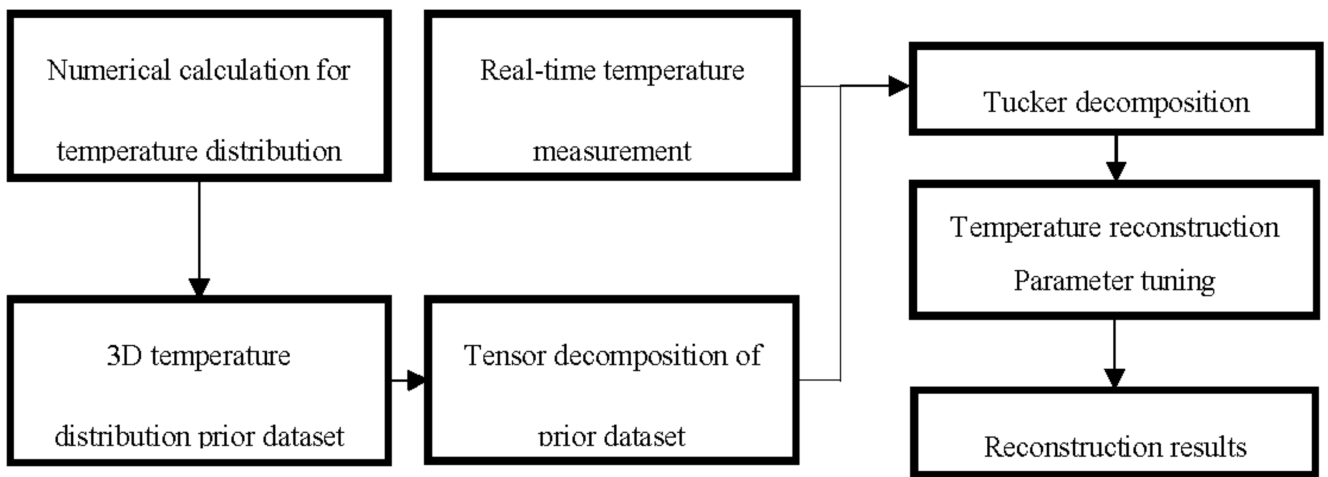

Figure 1. Flow diagram of three-dimensional reconstruction method.

\section{Numerical Simulation and Analysis}

On the basis of the previous research, in order to reduce the computational cost, numerical simulation is used to quantitatively demonstrate the feasibility of the proposed algorithm based on the Tucker decomposition and determines the effectiveness of the method.

A space of $4 \mathrm{~m} \times 4 \mathrm{~m} \times 4 \mathrm{~m}$ is constructed as a 3D temperature field reconstruction area. Set three different 3D temperature distribution numerical models as the research objects of the temperature reconstruction in this section, which are used to calculate the prior dataset and the test dataset. Apply the proposed 3D temperature distribution reconstruction algorithm to reconstruct the temperature profile under different test conditions. The effectiveness and feasibility of the reconstruction algorithm are analyzed. Restricted by the existing calculation conditions, the three-dimensional temperature distribution data is characterized by selecting 2D temperature field sections at different heights $(0.5 \mathrm{~m}, 1.5 \mathrm{~m}$, $2.5 \mathrm{~m}$ and $3.5 \mathrm{~m})$.

\subsection{Single-Peak 3D Temperature Distribution Reconstruction}

Set the single-peak 3D temperature distribution model as follows:

$$
T_{1}(x, y)=600+\frac{a}{(x-2)^{2}+(y-1)^{2}+(z-2.5)^{2}+1},
$$

where $T_{1}(x, y)$-temperature at the points $(x, y, z)$ of the single-peak 3D temperature distribution $(\mathrm{K}) ; x-\mathrm{X}$ coordinate $(\mathrm{m}) ; y-\mathrm{Y}$ coordinate $(\mathrm{m}) ; z-\mathrm{Z}$ coordinate $(\mathrm{m})$ and $a-$ initial boundary condition.

In the reconstruction area, use Equation (19) to calculate the temperature value. In order to reduce the amount of calculation, the $X$-axis and $Y$-axis directions take $0.1 \mathrm{~m}$ as the step length, and the Z-axis direction takes $1 \mathrm{~m}$ as the step length to start sampling from the origin. To form 3D temperature distribution data, $4 \times 40 \times 40$ temperature values are collected. Calculate the temperature field under different boundary conditions, construct a priori dataset with dimensions of $30 \times 4 \times 40 \times 40$ and test datasets with dimensions of $5 \times 4 \times 40 \times 40$. Set the prior dataset and the test dataset of the single-peak 
3D temperature distribution model and the boundary conditions as shown in Appendix A Tables A1 and A2.

According to the $3 \mathrm{D}$ temperature distribution reconstruction calculation process, the prior dataset is decomposed by the Tucker decomposition algorithm to obtain the reconstruction coefficient matrix. Select 16 coordinate points as temperature measuring points to reconstruct the $3 \mathrm{D}$ temperature profile.

The temperature distribution of test condition 5 calculated is shown in Figure 2.

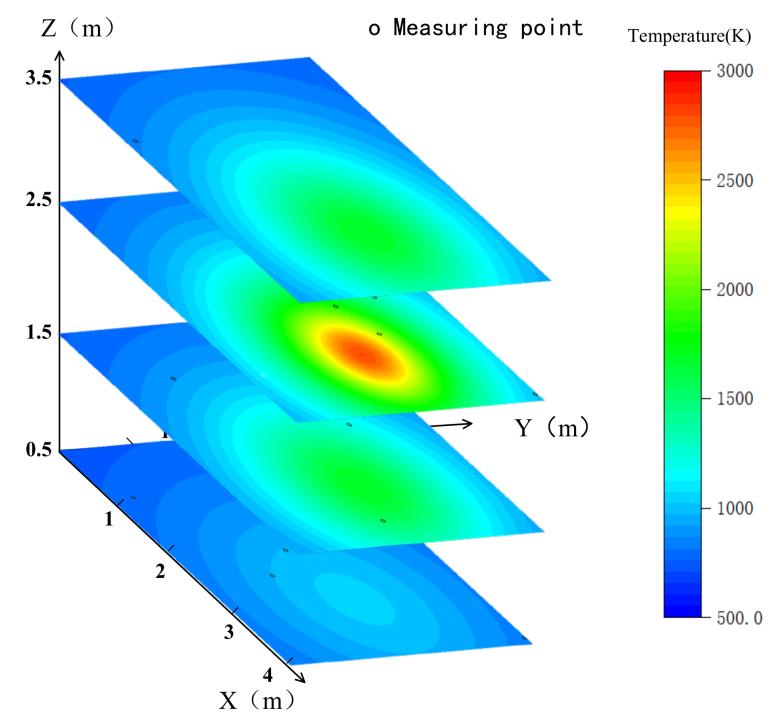

Figure 2. Calculation results of single-peak temperature field model in a cubic area.

The temperature field result of the test condition 5 obtained by the Tucker algorithm is as follows.

The boundary condition of test condition 5 is outside the constraint conditions of the priori dataset. As shown in Figure 3, the proposed algorithm still realizes the effective reconstruction.

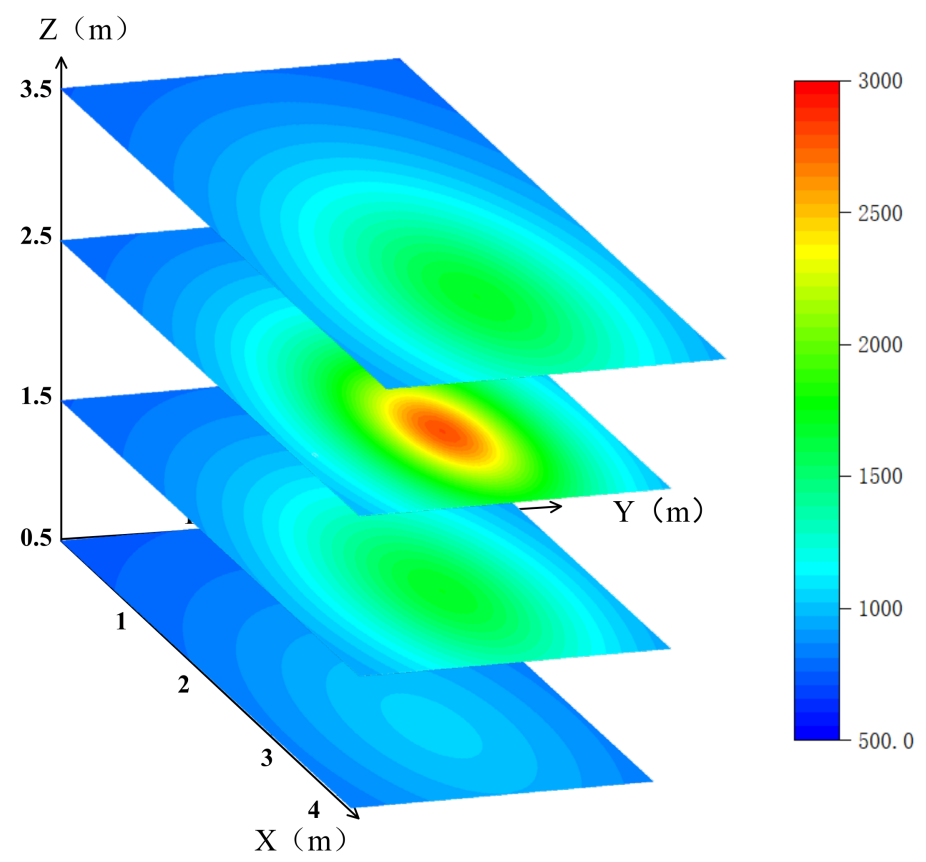

Figure 3. Reconstruction result of single-peak temperature field model in cubic area. 
To analyze the adaptability of the 3D temperature distribution reconstruction algorithm under different working conditions, the temperature profile data of the five test working conditions are reconstructed, and the reconstruction error is shown in Figure 4 below.

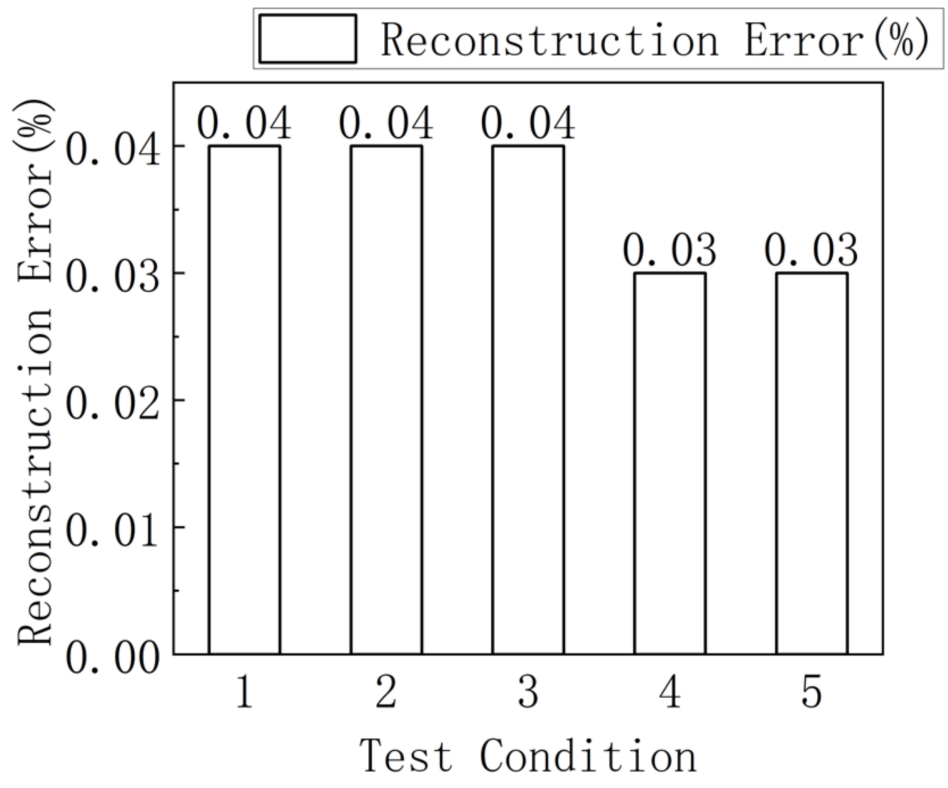

Figure 4. Reconstruction errors of single-peak temperature field model in cubic area.

As shown in Figure 4, the temperature field reconstruction results under five test conditions are analyzed. It can be seen that in the construction of single-peak 3D temperature distribution, the three-dimensional temperature field reconstruction algorithm based on the Tucker decomposition can effectively reconstruct the temperature profile data under different conditions.

\subsection{Two-Peak 3D Temperature Distribution Reconstruction}

Set two-peak 3D temperature distribution as follows:

$$
T_{2}(x, y)=200+\frac{a}{(x-2)^{2}+(y-1)^{2}+(z-1)^{2}+1}+\frac{b}{(x-3)^{2}+(y-2)^{2}+(z-1.5)^{2}+1}
$$

where $T_{2}(x, y)$-points $(x, y, z)$ of two-peak 3D temperature distribution model $(\mathrm{K})$; and $a, b$-initial boundary conditions.

Set the prior dataset and test dataset of the two-peak three-dimensional temperature distribution model and the boundary conditions as shown in Appendix A Tables A3 and A4.

The data acquisition and reconstruction process of the two-peak temperature distribution model is consistent with the calculation procedure of the single-peak mentioned above. The calculated temperature distribution of test condition 5 and the reconstruction result of the temperature field of test condition 5 calculated by the algorithm are shown in Figure 5 . 


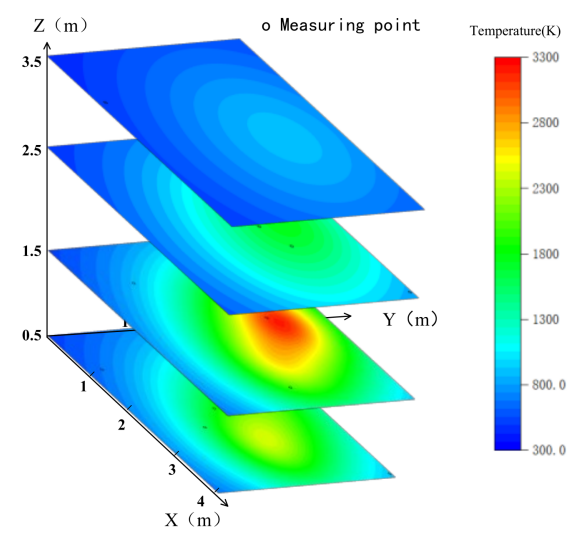

(a)

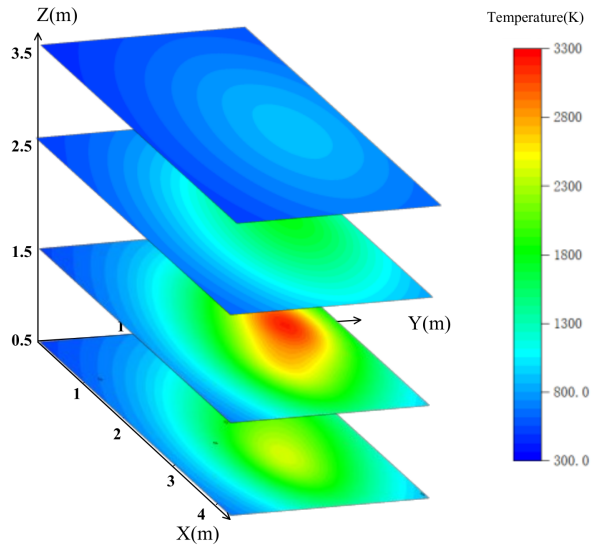

(b)

Figure 5. Two-peak temperature distribution model in cubic area: (a) calculation results of two-peak temperature distribution model in a cubic area; (b) reconstruction result of two-peak temperature distribution model in cubic area.

The 3D temperature field reconstruction algorithm is used to reconstruct the temperature distribution data of the five test conditions. The error is shown in Figure 6.

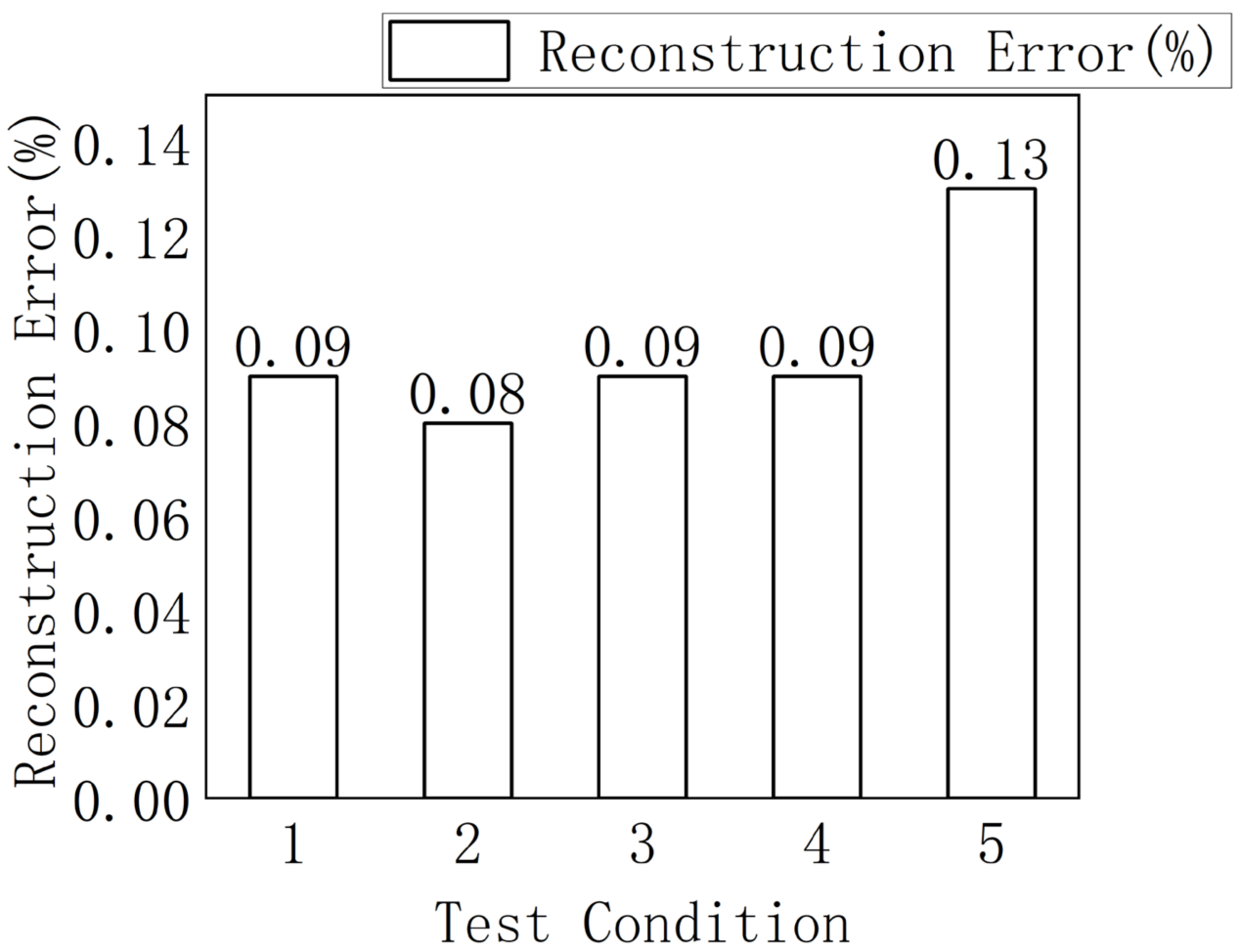

Figure 6. Reconstruction errors of two-peak temperature distribution model in cubic area.

Analysis of Figures 5 and 6 shows that in the five test conditions of the double-peak $3 \mathrm{D}$ temperature distribution model, the proposed algorithm can effectively realize the temperature distribution reconstruction.

\subsection{Three-Peak 3D Temperature Distribution Reconstruction}

Set the three-peak 3D temperature distribution as follows: 


$$
T_{3}(x, y)=270+\frac{a}{(x-0.5)^{2}+(y-1)^{2}+(z-1)^{2}+1}+\frac{b}{(x-3)^{2}+(y-2)^{2}+(z-1.5)^{2}+1}+\frac{b}{(x-2)^{2}+(y-2)^{2}+(z-2)^{2}+1}
$$

where $T_{3}(x, y)$-points $(x, y, z)$ of three-peak 3D temperature distribution model (K).

Set the model prior dataset and test dataset boundary conditions as shown in Appendix A Tables A5 and A6.

The calculated temperature distribution of test condition 5 and the reconstruction result of the temperature field of test condition 5 calculated by the algorithm are shown in Figure 7 below.

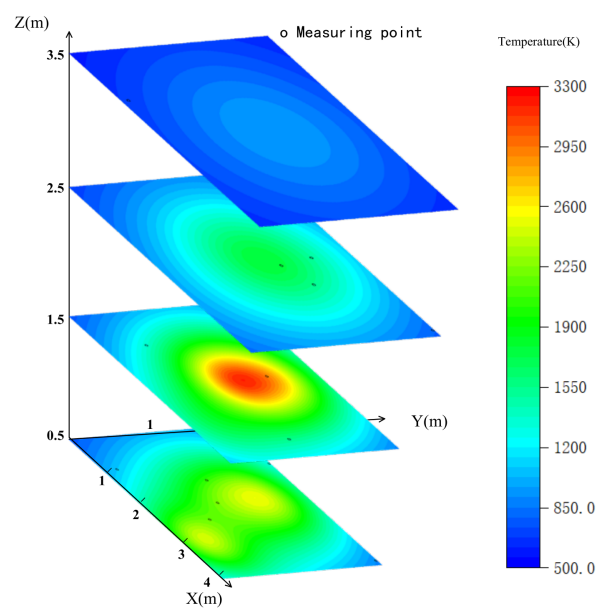

(a)

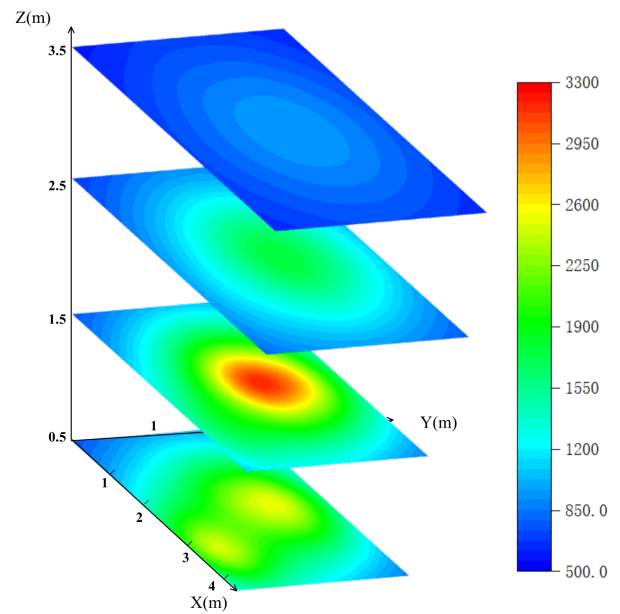

(b)

Figure 7. Three-peak temperature distribution model in a cubic area: (a) calculation results of three-peak temperature distribution model in a cubic area; (b) reconstruction results of three-peak temperature distribution model in a cubic area.

The Tucker decomposition algorithm is used to reconstruct the temperature distribution data of the five test conditions. The error is shown in Figure 8.

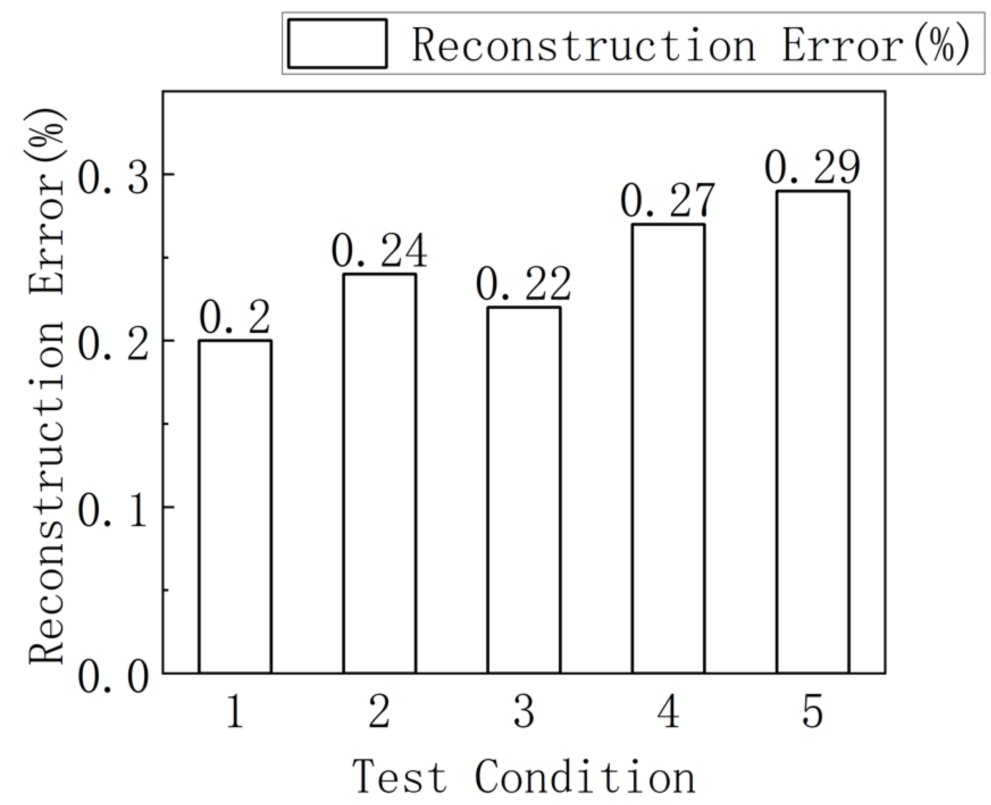

Figure 8. Reconstruction errors of three-peak temperature distribution model in cubic area. 
An analysis of Figures 7 and 8 shows that in the five test conditions of the three-peak 3D temperature distribution model, the 3D temperature distribution reconstruction algorithm proposed in this paper can effectively realize the temperature distribution reconstruction.

Based on the above analysis, this section uses the calculation data of single-peak, two-peak and three-peak three-dimensional temperature distribution models to verify the effectiveness and practicability of the proposed algorithm. The reconstruction results illustrate the feasibility of applying Tucker decomposition algorithm to the 3D temperature distribution reconstruction.

\section{Simulation Experiment}

Based on the preliminary demonstration of the effectiveness of the algorithm, in order to analyze the application effect of the reconstruction algorithm in the combustion, this paper takes the Flame D in Sandia laboratory as the research object and builds a numerical simulation model of methane combustion. The practicability of the reconstruction algorithm is analyzed by the simulation calculation data, and the validity of the temperature distribution reconstruction algorithm proposed in this paper is further demonstrated.

\subsection{Methane Burning Duty Flame}

The duty flame is a small flame set around the high-speed fuel outlet of the burner to achieve ignition and stable combustion. At Sandia Laboratories, Flame D is a typical jet flame obtained by burning at initial conditions with a methane inlet velocity of $49.6 \mathrm{~m} / \mathrm{s}$ and a hot air velocity of $11.4 \mathrm{~m} / \mathrm{s}$ formed by the duty flame [32,33].

Sandia Labs has measured the flame burning area of Flame D through laser-induced fluorescence, and the measurement data has been made public. The burner outlet structure used in Flame D is shown in Figure 9.

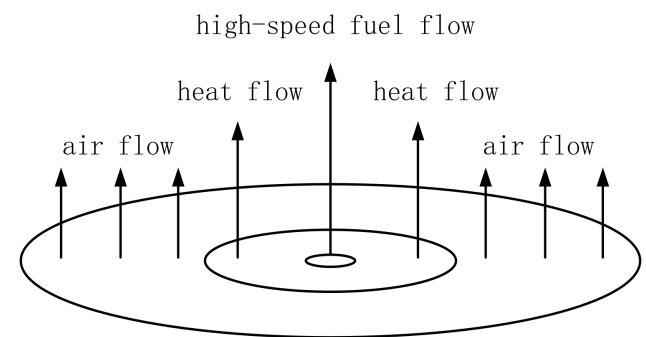

Figure 9. Diagram of burner outlet structure.

The geometric dimensions of the burner outlet are as follows: the diameter of the fuel outlet is $\mathrm{d}=7.2 \mathrm{~mm}$; the diameter of the wake heat flow outlet is $18.2 \mathrm{~mm}$, which is an annular structure; the outside of the heat flow outlet is air flow. In order to facilitate the establishment of the model in the subsequent process, the diameter $d$ of the fuel outlet is used as the unit length to characterize the size and coordinate position [33].

The fuel consists of methane and air mixed in a volume ratio of 1:3. The accompanying heat flow is formed by the combustion of mixed gases such as acetylene and hydrogen. The specific parameter data are shown in Table 1.

\subsection{Numerical Simulation Model Settings}

Flame D is a typical turbulent jet flame. The Reynolds number of the flame is 22,400, and the fuel inlet velocity is relatively fast, which belongs to the fully developed fast turbulent combustion process. The Realizable model is used in the simulation calculation. According to the actual parameters, the following settings are made: a cylindrical area with a diameter of $40 \mathrm{~d}$ and a height of $80 \mathrm{~d}$ is designated as the calculation area, and it is divided into 1,823,760 grids. The geometry of the Flame D combustion simulation model is shown in Figure 10. 
Table 1. Parameters of Flame D [32].

\begin{tabular}{cccc}
\hline Parameter Name & Fuel & Heat Flow & Air \\
\hline Velocity (m/s) & 49.6 & 11.4 & 0.9 \\
Pressure (atm) & 0.993 & 0.993 & 0.993 \\
Temperature (K) & 294 & 1880 & 291 \\
& & $\mathrm{~N}_{2}: 0.7342$ & $\mathrm{O}_{2}: 0.054$ \\
Component Proportion(\%) & $\mathrm{CH}_{4}: 0.1575$ & $\mathrm{H}_{2} \mathrm{O}: 0.0942$ & \\
& air: 0.1575 & $\mathrm{CO}_{0}: 00407$ & $\mathrm{CO}_{2}: 0.1098$ \\
\hline
\end{tabular}

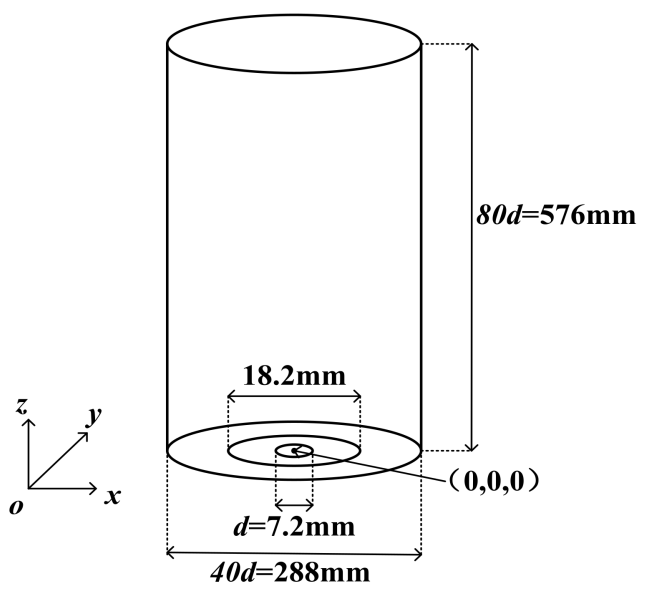

Figure 10. Geometry of the simulation model.

In order to ensure that the numerical simulation model can accurately simulate the Flame D combustion process, the parameters of the model are set based on the actual operating conditions, and appropriate optimization is carried out on the basis of taking into account the calculation efficiency and accuracy.

\subsection{Numerical Simulation Calculation Results Analysis}

Sandia Labs has released a measurement data set of Flame D, taking the cross-sections formed by $+\mathrm{z}$ and $+\mathrm{x}$ in the model as the research object. Compare the simulation data with the measured data to analyze the accuracy of the simulation.

The comparison between the simulation calculation data of the $z$-axis observation point and the temperature measurement data of the corresponding position is shown in the following figure.

It can be seen from Figure 11 that there is a certain deviation between the simulation results and the measured data. Analyzing the influencing factors, the error between the calculation result and the measured data mainly comes from the solution process and the model building process. Since the grid independence analysis has been carried out, the influence of grid division on the calculation results can be ignored, so the error in the solution process mainly comes from the discrete process of the governing equations. In the process of model establishment, the selection of turbulence model, chemical reaction model and parameter setting, as well as the simplification and assumption in the process of model building, are the main factors that cause the deviation between the calculated results and the measured data. 


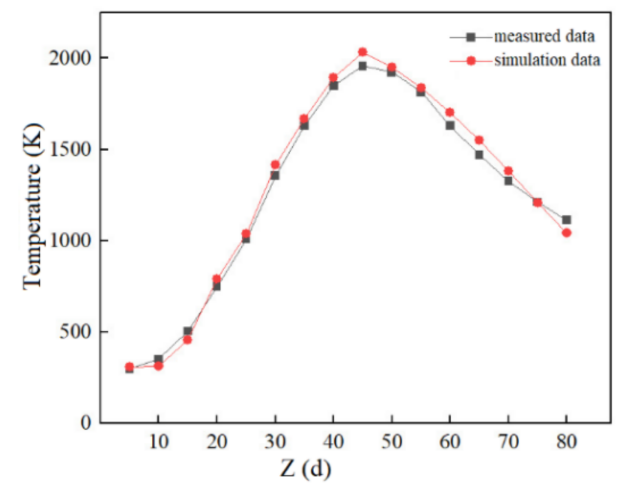

Figure 11. Temperature comparison in z-axis.

Nevertheless, as shown in Figure 11, the temperature calculated values at different positions are basically consistent with the general trend of the actual temperature distribution, that is, the simulation calculation results are close to the actual measured values.

In addition, the $z$-axis coordinates are taken as $30 \mathrm{~d}, 45 \mathrm{~d}, 60 \mathrm{~d}$ and $75 \mathrm{~d}$ for the radial observation point temperature data comparison as follows.

Similar to the conclusion in Figure 11, the calculated temperature values at different radial positions shown in Figure 12 are basically consistent with the overall trend of the actual temperature distribution. Therefore, the simulation model can better simulate the temperature distribution of Flame D.

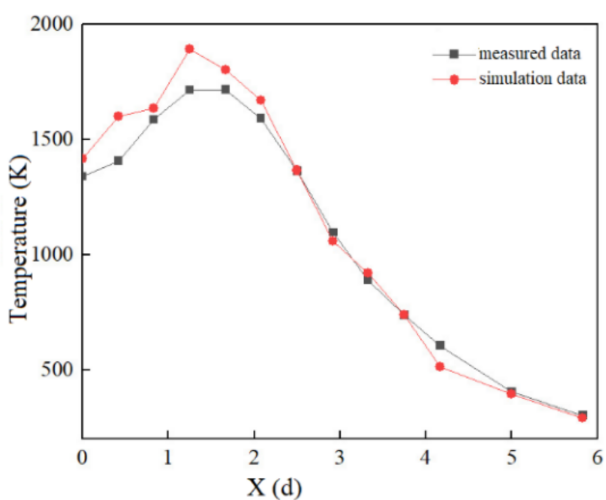

(a)

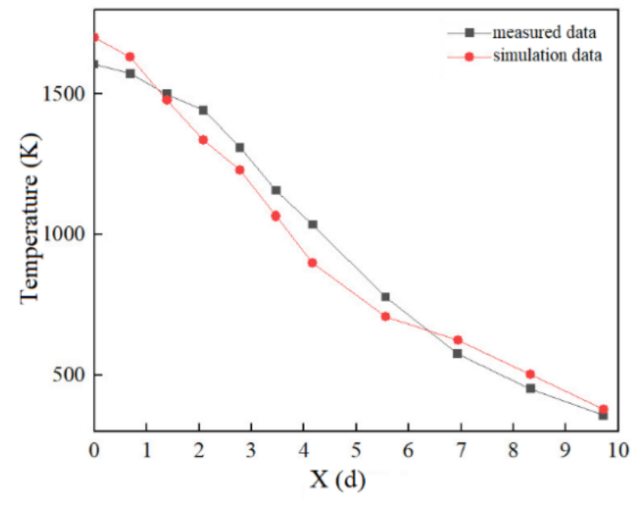

(c)

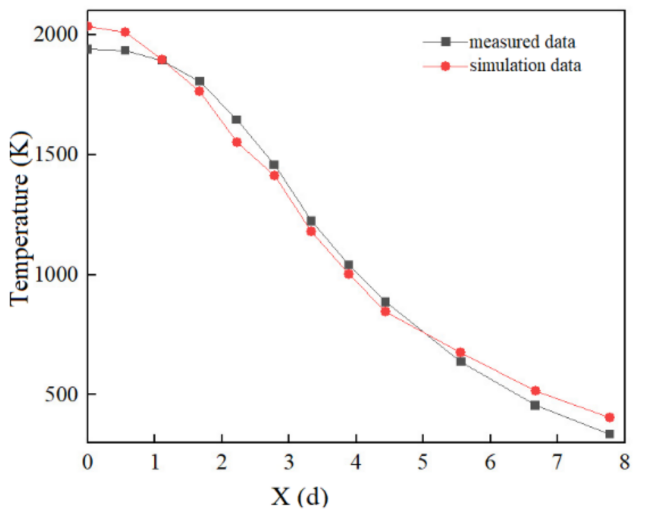

(b)

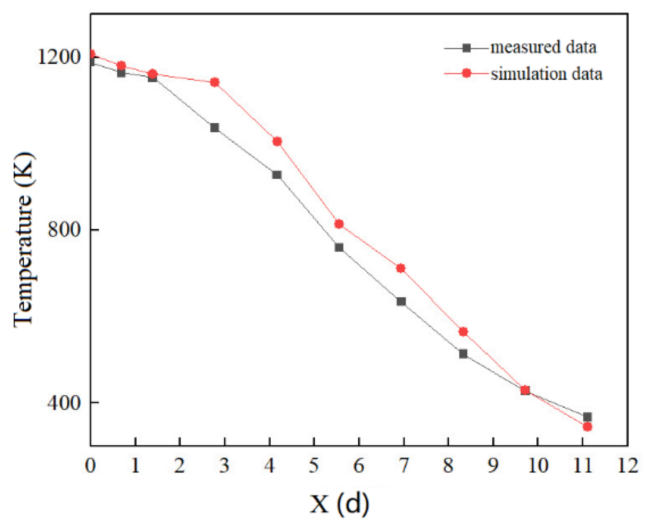

(d)

Figure 12. Temperature comparison in different locations: (a) Temperature comparison in $\mathrm{z}=30 \mathrm{~d}$; (b) Temperature comparison in $\mathrm{z}=45 \mathrm{~d}$; (c) Temperature comparison in $\mathrm{z}=60 \mathrm{~d}$; (d) Temperature comparison in $\mathrm{z}=75 \mathrm{~d}$. 
In summary, although the numerical calculation method has approximations and assumptions that cannot be avoided in the process of solving and model building, it can still accurately simulate the basic properties of the simulation object and meet the actual needs of engineering applications. The methane combustion simulation model established in this paper can simulate the real temperature distribution of Flame D accurately.

\subsection{Prior Data and Test Data Acquisition}

As mentioned in the previous section, the established numerical simulation model can simulate the methane combustion process, and the calculated temperature distribution data is close to the actual value and can reflect the distribution of the actual combustion temperature. Therefore, this model can be applied to build prior datasets as well as the test dataset for the temperature distribution reconstruction. Considering the turbulence intensity and the range of the prior dataset, the boundary conditions for methane combustion simulation calculation are set as shown in Table A7.

In order to construct the temperature distribution reconstruction calculation samples, five test cases were set up. The boundary conditions of the test condition are different from the prior condition, and the boundary condition of the test condition 5 is set outside the range of the boundary condition of the prior condition to test the robustness of the temperature distribution reconstruction algorithm. The boundary condition settings of the test conditions are shown in Table 2.

Table 2. Boundary conditions of combustion test dataset.

\begin{tabular}{ccc}
\hline Condition Name & $\begin{array}{c}\text { Fuel Velocity } \\
(\mathbf{m} / \mathbf{s})\end{array}$ & $\begin{array}{c}\text { Heat Flow } \\
\text { Velocity } \\
(\mathbf{m} / \mathbf{s})\end{array}$ \\
\hline Test Condition 1 & 32 & 10.6 \\
Test Condition 2 & 49 & 15.3 \\
Test Condition 3 & 57 & 17.7 \\
Test Condition 4 & 68 & 14.4 \\
Test Condition 5 & 77 & 26 \\
\hline
\end{tabular}

\subsection{Simulation Data Reconstruction Analysis}

The feasibility of the reconstruction algorithm is verified by using the temperature distribution data calculated from the numerical simulation model.

The temperature of 1600 locations on the three planes of $\mathrm{z}=30 \mathrm{~d}, 45 \mathrm{~d}$ and $60 \mathrm{~d}$ of the methane combustion simulation model were selected as the research objects of the three-dimensional temperature distribution reconstruction. The selected positions include 36 measuring points on the axial $30 \mathrm{~d}, 45 \mathrm{~d}$, and $60 \mathrm{~d}$ published by Sandia Labs so as to facilitate the experimental data reconstruction and analysis. Based on the simulation calculation results of the prior and test conditions, a three-dimensional temperature distribution reconstruction prior dataset with dimensions of $24 \times 3 \times 40 \times 40$ and a test dataset with dimensions of $5 \times 3 \times 40 \times 40$ were constructed.

The 3-D temperature distribution reconstruction algorithm is used to reconstruct the test conditions' temperature distribution. The measurement data is taken from the simulation calculation data. Based on the measurement matrix, the temperature of the corresponding position in the test dataset is extracted as the temperature measurement data, which is used for the temperature distribution reconstruction.

Taking the test case 5 as an example, the simulation results of the temperature distribution in the three target sections are shown in Figure 13. 


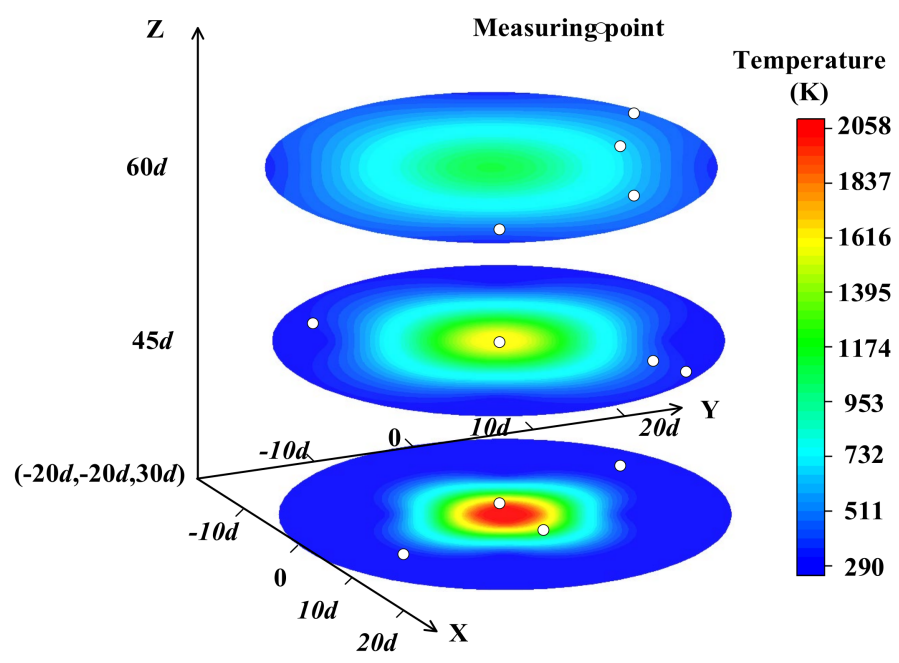

Figure 13. Simulation results of test case 5 (Three-dimensional).

Figure 13 shows the simulation results of the three-dimensional temperature distribution calculated by using the simulation numerical software according to the geometric parameters of the established model, combustion parameters and boundary conditions of test condition 5. The white points in the Figure 13 represent the arrangement of temperature measuring points.

The temperatures of the 12 measuring points shown in Figure 13 are used as temperature measurement data, and the reconstruction calculation is performed using the core tensor with dimensions of $1 \times 2 \times 2 \times 3$.

After reconstruction calculation, it can be seen that the reconstruction error of test case 5 is $4.13 \%$, and the temperature deviation of the reconstruction results is less than $90 \mathrm{~K}$, which realizes the effective original temperature distribution reconstruction and verifies the 3-D temperature distribution reconstruction algorithm.

In order to better show the reconstruction results, the 3D temperature distribution reconstruction calculation and analysis process presents the reconstruction effect as a contour map of the absolute value of the deviation between the reconstructed temperature value and the calculated temperature value. The absolute value of the temperature deviation between the reconstruction calculation results and the simulation calculation results is shown in Figure 14.

In order to analyze the adaptability of the reconstruction algorithm under different working conditions, the three-dimensional temperature distribution reconstruction algorithm was used to reconstruct the five test conditions' data, and the reconstruction errors are shown in Figure 15.

As shown in Figures 14 and 15, the three-dimensional temperature distribution reconstruction algorithm can effectively reconstruct the five different test conditions' data, which further verifies the practicability and effectiveness. 


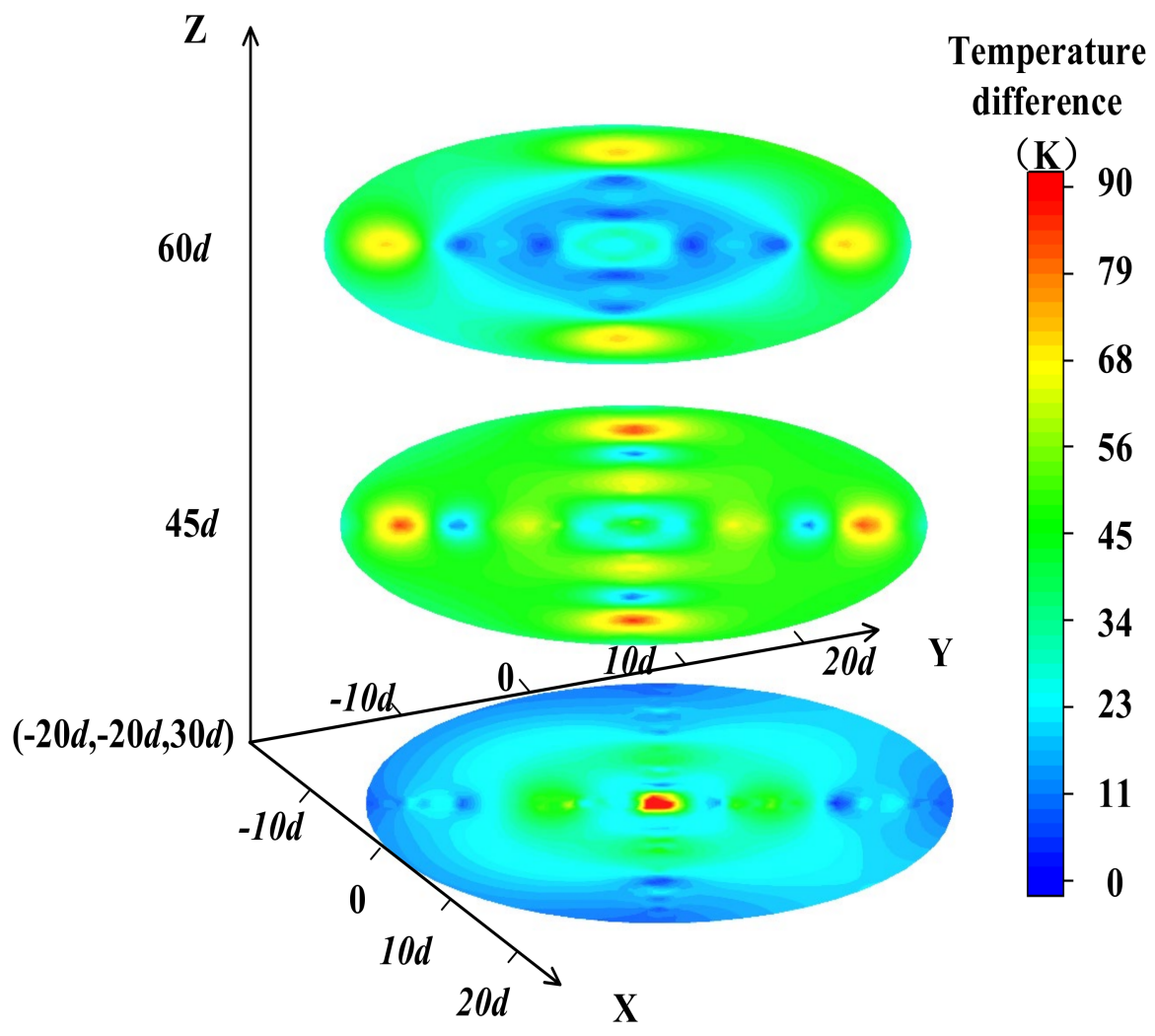

Figure 14. Temperature deviations of reconstruction results in test case 5 (Three-dimensional).

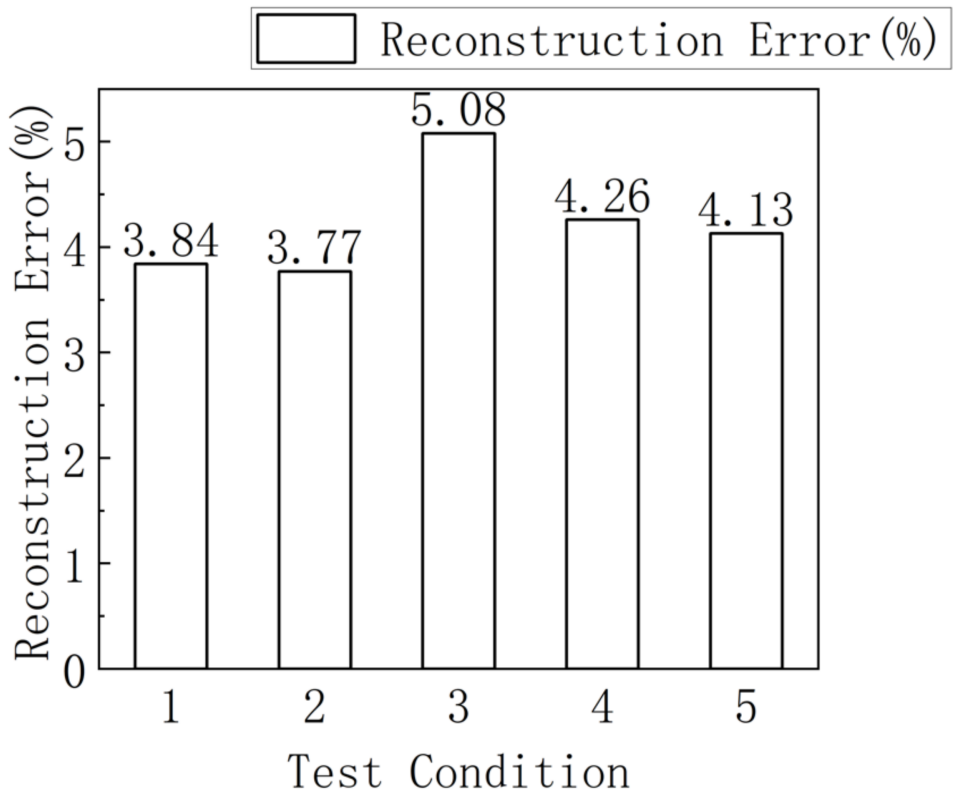

Figure 15. Reconstruction errors of simulation data (Three-dimensional).

\section{Effect of Core Tensor Dimension on Reconstruction Accuracy}

On the basis of the validity of the reconstruction algorithm, the influence of the core tensor dimension on the reconstruction accuracy during the temperature distribution reconstruction is analyzed to further improve the algorithm accuracy. The temperatures of 1600 positions were selected on the three planes of $z=30 d, 45 d$ and $60 \mathrm{~d}$ in the 
methane combustion simulation model as the research object of 3D temperature distribution reconstruction.

The three-dimensional temperature distribution reconstruction algorithm based on Tucker decomposition uses the key information of the prior dataset carried by the core tensor to realize the reconstruction. The core tensor of the prior dataset has four dimensions, so it is necessary to analyze the values of the four dimensions one by one.

The prior dataset of 3D temperature distribution reconstruction is a tensor of $24 \times 3 \times 40 \times 40$. The dimension in the 2-modular direction in the prior data is small, so the calculation amount of optimizing the dimension value in the 2-modular direction is also small. Therefore, the effect of the core tensor dimension on the reconstruction accuracy is explored from the 2-modular direction.

Set up the 2-module direction dimension influence analysis experiment, and the experimental steps are as follows:

(1) In order to avoid too few measurement data points affecting the analysis results, 50 measurement data points were used in the reconstruction. In order to avoid the influence of the dimension of other modes, the dimension of other modes is fixed to 10;

(2) Perform Tucker decomposition on the prior dataset to obtain a core tensor with a set dimension of $10 \times n \times 10 \times 10, n=1,2,3$;

(3) Use the core tensor, decomposition factor matrix and measured temperature to reconstruct the 3D temperature field, and calculate the reconstruction error. Add noise data with $\mathrm{SNR}=40 \mathrm{~dB}$ to the measured temperature and compare the reconstruction results in the presence of measurement error;

(4) Repeat the above steps 1000 times to obtain the average reconstruction error in order to eliminate the influence of randomness in the temperature measurement so a universal conclusion can be obtained;

(5) Repeat the above steps until the dimension $n$ has traversed all the values.

Taking the test case 5 as an example, the different 2-mode dimensions reconstruction results are shown in Table 3.

Table 3. Reconstruction errors with different dimensions (Mode-2).

\begin{tabular}{ccc}
\hline Dimension & $\begin{array}{c}\text { Raw Data Reconstruction } \\
\text { Error (\%) }\end{array}$ & $\begin{array}{c}\text { Noise-Added Data } \\
\text { Reconstruction Error (\%) }\end{array}$ \\
\hline 1 & 18.21 & 20.05 \\
2 & 4.2 & 9.33 \\
3 & 0.18 & 7.94 \\
\hline
\end{tabular}

As can be seen from Table 3, as the dimensionality in the 2-mode direction increases, the reconstruction error decreases whether using the original temperature measurement data or using the noise-added measurement data. Therefore, the 2-modular dimension of the core tensor is set to be three in the follow-up research work.

When the 2-module dimension is three, the effect of the 1-module dimension on the reconstruction accuracy is analyzed. The 3-module and 4-module dimension are set to 10 , and the value range of the 1-module dimension $n$ is 1 to 10 . Other parameters and calculation steps are consistent with the above analysis and will not be repeated here.

Taking the test case 5 as an example, the reconstruction results of different 1-module dimensions are shown in the table below:

In Table 4, it can be seen that the reconstruction error using the original temperature measurement data will gradually decrease with the dimension increase; the reconstruction error using noise-added measurement data increases with the increase of dimension. From the perspective of balancing the reconstruction accuracy and noise sensitivity, the 1-module dimension of the core tensor is set to two in the subsequent reconstruction work. Similarly, 
it is determined that the 3-module dimension is five, and the 4-module dimension is two. Finally, the optimized core tensor dimension is determined to be $2 \times 3 \times 5 \times 2$.

Table 4. Reconstruction errors with different dimension (Mode-1).

\begin{tabular}{ccc}
\hline Dimension & $\begin{array}{c}\text { Raw Data Reconstruction } \\
\text { Error (\%) }\end{array}$ & $\begin{array}{c}\text { Noise-Added Data } \\
\text { Reconstruction Error (\%) }\end{array}$ \\
\hline 1 & 2.63 & 2.63 \\
2 & 1.48 & 2.63 \\
3 & 1.33 & 3.41 \\
4 & 0.52 & 4.11 \\
5 & 0.53 & 4.6 \\
6 & 0.52 & 5.47 \\
7 & 0.2 & 6.61 \\
8 & 0.2 & 7.13 \\
9 & 0.18 & 7.23 \\
10 & 0.18 & 7.98 \\
\hline
\end{tabular}

The optimized core tensor dimension is used to add noise with different signal-tonoise ratios to Test Condition 5, and the comprehensive performance of the optimization algorithm under different noise conditions is investigated, as shown in Figure 16.

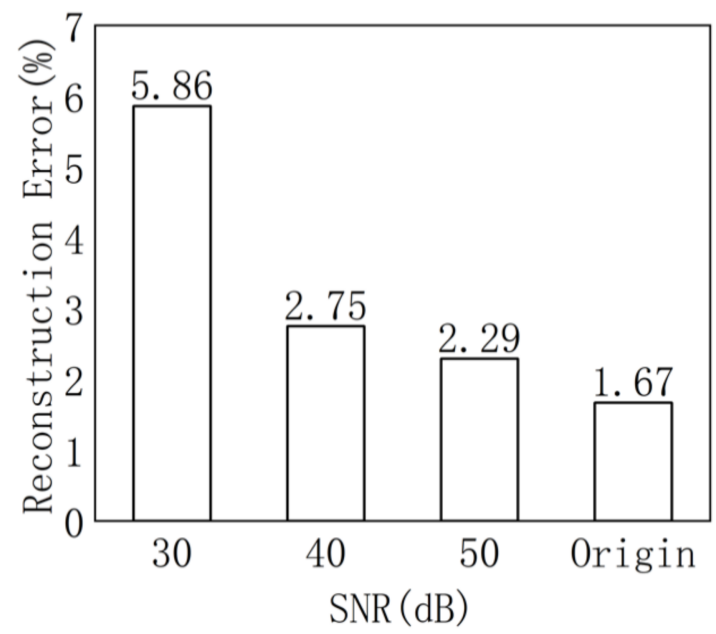

Figure 16. Sensitivity of optimization algorithm under different noise-added conditions.

The data of five test cases are reconstructed, and the reconstruction effect with $\mathrm{SNR}=40 \mathrm{~dB}$ noise-added is analyzed. The results are shown in Figure 17.

It can be seen from Figures 16 and 17 that the optimization effect of the core tensor dimension is stable in the five test conditions. Compared with the reconstruction error, the optimization algorithm can reduce the reconstruction error under different test conditions, and the optimization effect is more obvious with the increase of noise intensity. The optimized reconstruction algorithm has a high reconstruction accuracy and good anti-noise performance. 


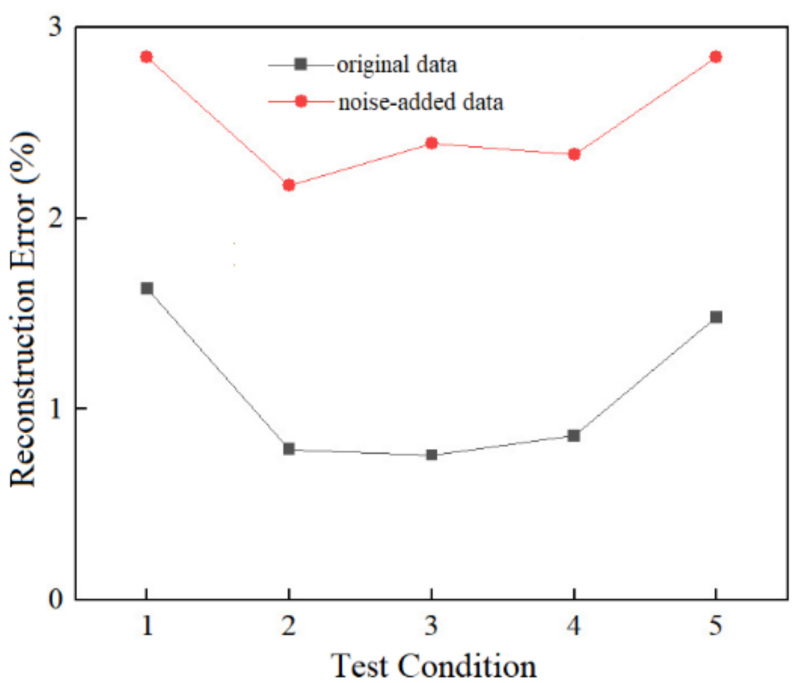

Figure 17. Optimized effects of core tensor dimension.

\section{Conclusions}

In this paper, a fast and accurate optimization algorithm based on Tucker decomposition has been proposed to reconstruct 3D temperature fields with sparse data. The relevant mathematical derivations from Tucker decomposition have been carried out to determine a $3 \mathrm{D}$ temperature field reconstruction algorithm. The dataset used to generate the core tensor plays a very important role in this method. When using CFD results to establish datasets, it is essential to sample large-scale boundary conditions that can cover most of the working conditions in the flow field. Simulations were performed, and numerical results demonstrated that the Tucker decomposition algorithm combined with sparse measurement is capable of reconstructing the 3D temperature field quickly and accurately. To evaluate the performance of this algorithm, a numerical simulation model of methane combustion has been established based on the Flame D of Sandia Laboratory. Simulation experimental results showed that this method can reconstruct temperature distribution rapidly using limited measurement data with the relative of less than $5.08 \%$, which verifies the feasibility of this algorithm. It can greatly reduce the computational complexity and measurement cost. Finally, this paper analyzes the influence of core tensor dimension on reconstruction accuracy in the process of a temperature distribution reconstruction calculation. Core tensor can be extracted from a high-dimensional experience dataset. The first few dimensions carry most of the useful information. With the use of a higher dimension core vector, too much useless information is introduced to the reconstruction process, and the reconstruction results are more sensitive to the noise. Thus, it is necessary to obtain a balance between reconstruction accuracy and noise sensitivity. The results show that the reconstruction error is less than $2 \%$ by optimizing the core tensor dimension.

Furthermore, the present study lays the groundwork for future research into some interesting fields with several objectives, including the following:

1. In order to show more object field characteristics, this study uses a wide range of boundary conditions to establish the dataset. This helps to generate the core tensor of Tucker decomposition, but how to construct dataset more effectively remains to be solved. Further research can focus on the source of empirical data, effective dataset sampling and the impact of dataset noise.

2. The proposed method needs little measurement data to yield an acceptable reconstruction result. The sensor number has some effect on the reconstruction result: the greater the number of sensors used, the better result. Therefore, it is necessary to explore the number of appropriate sensors needed to balance the measurement cost and reconstruction accuracy. 
3. When only a few sensors are used for reconstruction, sensor placement has a great influence on reconstruction accuracy. Testing the feasibility of finding a sensor placement optimization algorithm that can yield a much better reconstruction result.

Author Contributions: Conceptualization, Z.L. and S.L.; methodology, M.C.; software, P.Y.; validation, Z.L., M.C., Y.Z. and P.Y.; formal analysis, Z.L.; data curation, Z.L. and M.C.; writing-original draft preparation, Z.L.; writing-review and editing, Y.Z.; supervision, S.L.; project administration, S.L.; funding acquisition, S.L. All authors have read and agreed to the published version of the manuscript.

Funding: This work was supported by the National Natural Science Foundation of China [grant number 61871181].

Data Availability Statement: Not applicable.

Conflicts of Interest: The authors declare no conflict of interest.

\section{Nomenclature}

\begin{tabular}{|c|c|}
\hline & Indices \\
\hline$\chi$ & N-order tensors \\
\hline$\varepsilon$ & N-order error tensor \\
\hline$N$ & the decomposition factor \\
\hline$U^{(n)}$ & the factor matrix \\
\hline$\zeta$ & tensor $\chi$ Tucker decomposition core tensor \\
\hline$\times_{N}$ & tensor $\chi \mathrm{N}$-mode product \\
\hline$U^{(N)}$ & tensor $\chi$ decomposition factor matrix in the $\mathrm{N}$-module direction \\
\hline$T R$ & a fourth-order tensor of the prior data \\
\hline$C_{t}$ & the core tensor of prior data set tensor TR Tucker decomposition \\
\hline$F_{t(i)}$ & decomposition factor matrix in the i-module direction of tensor TR \\
\hline $\mathrm{P}$ & the decomposition factor matrix in the 1-mode direction \\
\hline Q & the decomposition factor matrix in the 2-mode direction \\
\hline$f_{1 i}$ & $\begin{array}{l}\text { the } i \text { th factor vector in the decomposition factor matrix in the direction of } \\
1 \text { modulus }\end{array}$ \\
\hline$A$ & reconstruction coefficient tensor \\
\hline$f_{1 x}$ & $\begin{array}{l}\text { the three-dimensional temperature distribution to be solved to reconstruct } \\
\text { the 1-mode decomposition factor vector }\end{array}$ \\
\hline$t_{x}$ & first-order representation of three-dimensional temperature distribution \\
\hline$A_{1 \text { unfold }}$ & the expansion of the coefficient matrix A along the direction of 1 modulus \\
\hline$t_{M}$ & a vector of small number of temperature measurements \\
\hline$M$ & $\begin{array}{l}\text { the measurement matrix, which characterizes the location of the } \\
\text { measurement points }\end{array}$ \\
\hline$t_{M}$ & $\begin{array}{l}\text { obtained by measurement } \\
\text { Abbreviations }\end{array}$ \\
\hline PCA & principal component analysis \\
\hline POD & proper orthogonal decomposition \\
\hline HOSVD & higher-order singular value decomposition \\
\hline SVD & singular value decomposition \\
\hline
\end{tabular}




\section{Appendix A}

Table A1. Boundary conditions of prior dataset (single-peak model in a cubic area).

\begin{tabular}{cccc}
\hline $\begin{array}{c}\text { Working } \\
\text { Condition }\end{array}$ & Boundary Condition (a) & $\begin{array}{c}\text { Working } \\
\text { Condition }\end{array}$ & Boundary Condition (a) \\
\hline Condition 1 & 600 & Condition 16 & 1350 \\
Condition 2 & 650 & Condition 17 & 1400 \\
Condition 3 & 700 & Condition 18 & 1450 \\
Condition 4 & 750 & Condition 19 & 1500 \\
Condition 5 & 800 & Condition 20 & 1550 \\
Condition 6 & 850 & Condition 21 & 1600 \\
Condition 7 & 900 & Condition 22 & 1650 \\
Condition 8 & 950 & Condition 23 & 1700 \\
Condition 9 & 1000 & Condition 24 & 1750 \\
Condition 10 & 1050 & Condition 25 & 1800 \\
Condition 11 & 1100 & Condition 26 & 1850 \\
Condition 12 & 1150 & Condition 27 & 1900 \\
Condition 13 & 1200 & Condition 28 & 1950 \\
Condition 14 & 1250 & Condition 29 & 2000 \\
Condition 15 & 1300 & Condition 30 & 2050 \\
\hline
\end{tabular}

Table A2. Boundary conditions of test dataset (single-peak model in a cubic area).

\begin{tabular}{cc}
\hline Working Condition & Boundary Condition (a) \\
\hline Test Condition 1 & 723 \\
Test Condition 2 & 1107 \\
Test Condition 3 & 1426 \\
Test Condition 4 & 1846 \\
Test Condition 5 & 2200 \\
\hline
\end{tabular}

Table A3. Boundary conditions of prior dataset (Two-peak model in a cubic area).

\begin{tabular}{|c|c|c|}
\hline Wording Condition & Boundary Condition (a) & Boundary Condition (b) \\
\hline Condition 1 & 800 & 1000 \\
\hline Condition 2 & 800 & 1200 \\
\hline Condition 3 & 800 & 1400 \\
\hline Condition 4 & 800 & 1600 \\
\hline Condition 5 & 800 & 1800 \\
\hline Condition 6 & 1000 & 800 \\
\hline Condition 7 & 1000 & 1200 \\
\hline Condition 8 & 1000 & 1400 \\
\hline Condition 9 & 1000 & 1600 \\
\hline Condition 10 & 1000 & 1800 \\
\hline Condition 11 & 1200 & 800 \\
\hline Condition 12 & 1200 & 1000 \\
\hline Condition 13 & 1200 & 1400 \\
\hline Condition 14 & 1200 & 1600 \\
\hline Condition 15 & 1200 & 1800 \\
\hline Condition 16 & 1400 & 800 \\
\hline Condition 17 & 1400 & 1000 \\
\hline
\end{tabular}


Table A3. Cont.

\begin{tabular}{ccc}
\hline Wording Condition & Boundary Condition (a) & Boundary Condition (b) \\
\hline Condition 18 & 1400 & 1200 \\
\hline Condition 19 & 1400 & 1600 \\
\hline Condition 20 & 1400 & 1800 \\
\hline Condition 21 & 1600 & 800 \\
\hline Condition 22 & 1600 & 1000 \\
\hline Condition 23 & 1600 & 1200 \\
\hline Condition 24 & 1600 & 1400 \\
\hline Condition 25 & 1600 & 1800 \\
\hline Condition 26 & 1800 & 800 \\
\hline Condition 27 & 1800 & 1000 \\
\hline Condition 28 & 1800 & 1200 \\
\hline Condition 29 & 1800 & 1400 \\
\hline Condition 30 & 1800 & 1600 \\
\hline
\end{tabular}

Table A4. Boundary conditions of test dataset (Two-peak model in a cubic area).

\begin{tabular}{ccc}
\hline Working Condition & Boundary Condition (a) & Boundary Condition (b) \\
\hline Test Condition 1 & 823 & 1189 \\
Test Condition 2 & 1407 & 1147 \\
Test Condition 3 & 1426 & 1569 \\
Test Condition 4 & 1746 & 1601 \\
Test Condition 5 & 1983 & 2356 \\
\hline
\end{tabular}

Table A5. Boundary conditions of prior dataset (Three-peak model in a cubic area).

\begin{tabular}{ccc}
\hline Working Condition & Boundary Condition (a) & Boundary Condition (b) \\
\hline Condition 1 & 600 & 800 \\
\hline Condition 2 & 600 & 1000 \\
\hline Condition 3 & 600 & 1200 \\
\hline Condition 4 & 600 & 1400 \\
\hline Condition 5 & 600 & 1600 \\
\hline Condition 6 & 800 & 600 \\
\hline Condition 7 & 800 & 1000 \\
\hline Condition 8 & 800 & 1200 \\
\hline Condition 9 & 800 & 1400 \\
\hline Condition 10 & 800 & 1600 \\
\hline Condition 11 & 1000 & 600 \\
\hline Condition 12 & 1000 & 800 \\
\hline Condition 13 & 1000 & 1200 \\
\hline Condition 14 & 1000 & 1400 \\
\hline Condition 15 & 1000 & 1600 \\
\hline Condition 16 & 1200 & 600 \\
\hline
\end{tabular}


Table A5. Cont.

\begin{tabular}{ccc}
\hline Working Condition & Boundary Condition (a) & Boundary Condition (b) \\
\hline Condition 17 & 1200 & 800 \\
\hline Condition 18 & 1200 & 1000 \\
\hline Condition 19 & 1200 & 1400 \\
\hline Condition 20 & 1200 & 1600 \\
\hline Condition 21 & 1400 & 600 \\
\hline Condition 22 & 1400 & 800 \\
\hline Condition 23 & 1400 & 1000 \\
\hline Condition 24 & 1400 & 1200 \\
\hline Condition 25 & 1400 & 1600 \\
\hline Condition 26 & 1600 & 600 \\
\hline Condition 27 & 1600 & 800 \\
\hline Condition 28 & 1600 & 1000 \\
\hline Condition 29 & 1600 & 1200 \\
\hline Condition 30 & 1600 & 1400 \\
\hline
\end{tabular}

Table A6. Boundary conditions of test dataset (Three-peak model in a cubic area).

\begin{tabular}{ccc}
\hline Working Condition & Boundary Condition (a) & Boundary Condition (b) \\
\hline Test Condition 1 & 621 & 959 \\
Test Condition 2 & 1194 & 645 \\
Test Condition 3 & 1221 & 1369 \\
Test Condition 4 & 1577 & 1153 \\
Test Condition 5 & 1627 & 1773 \\
\hline
\end{tabular}

Table A7. Boundary conditions of combustion prior dataset.

\begin{tabular}{ccc}
\hline Condition Name & Fuel Velocity $(\mathbf{m} / \mathbf{s})$ & Heat Flow Velocity $(\mathbf{m} / \mathbf{s})$ \\
\hline Condition 1 & 25 & 10 \\
\hline Condition 2 & 25 & 14 \\
\hline Condition 3 & 25 & 18 \\
\hline Condition 4 & 25 & 22 \\
\hline Condition 5 & 35 & 10 \\
\hline Condition 6 & 35 & 14 \\
\hline Condition 7 & 35 & 18 \\
\hline Condition 8 & 35 & 22 \\
\hline Condition 9 & 45 & 10 \\
\hline Condition 10 & 45 & 14 \\
\hline Condition 11 & 45 & 18 \\
\hline Condition 12 & 45 & 22 \\
\hline Condition 13 & 55 & 10 \\
\hline Condition 14 & 55 & 14 \\
\hline Condition 15 & 55 & 18 \\
\hline
\end{tabular}


Table A7. Cont.

\begin{tabular}{ccc}
\hline Condition Name & Fuel Velocity $(\mathbf{m} / \mathbf{s})$ & Heat Flow Velocity $(\mathbf{m} / \mathbf{s})$ \\
\hline Condition 16 & 55 & 22 \\
\hline Condition 17 & 65 & 10 \\
\hline Condition 18 & 65 & 14 \\
\hline Condition 19 & 65 & 18 \\
\hline Condition 20 & 65 & 22 \\
\hline Condition 21 & 75 & 10 \\
\hline Condition 22 & 75 & 14 \\
\hline Condition 23 & 75 & 18 \\
\hline Condition 24 & 75 & 22 \\
\hline
\end{tabular}

\section{References}

1. Guan, Y.; He, W.; Murugesan, M.; Li, Q.; Liu, P.; Li, L.K. Control of self-excited thermoacoustic oscillations using transient forcing, hysteresis and mode switching. Combust. Flame 2019, 202, 262-275. [CrossRef]

2. Guan, Y.; Vikrant, G.; Karthik, K.; Li, L.K.B. Open-loop control of periodic thermoacoustic oscillations: Experiments and low-order modelling in a synchronization framework. Proc. Combust. Inst. 2018, 37, 5315-5323. [CrossRef]

3. Guan, Y.; Gupta, V.; Wan, M.; Li, L.K.B. Forced synchronization of quasiperiodic oscillations in a thermoacoustic system. J. Fluid Mech. 2019, 879, 390-421. [CrossRef]

4. Myung, C.L.; Ko, A.; Park, S. Review on characterization of nano-particle emissions and PM morphology from internal combustion engines: Part 1. Int. J. Automot. Technol. 2014, 15, 203-218. [CrossRef]

5. Myung, C.L.; Park, S. Exhaust nanoparticle emissions from internal combustion engines: A review. Int. J. Automot. Technol. 2012, 13, 9. [CrossRef]

6. Myung, C.L.; Lee, H.; Choi, K.; Lee, Y.J.; Park, S. Effects of gasoline, diesel, LPG, and low-carbon fuels and various certification modes on nanoparticle emission characteristics in light-duty vehicles. Int. J. Automot. Technol. 2009, 10, 537-544. [CrossRef]

7. Heeger, C.; Gordon, R.L.; Tummers, M.J.; Sattelmayer, T.; Dreizler, A. Experimental analysis of flashback in lean premixed swirling flames: Upstream flame propagation. Exp. Fluids 2010, 49, 853-863. [CrossRef]

8. Nauert, A.; Petersson, P.; Linne, M.; Dreizler, A. Experimental analysis of flashback in lean premixed swirling flames: Conditions close to flashback. Exp. Fluids 2007, 43, 89-100. [CrossRef]

9. Seffrin, F.; Fuest, F.; Geyer, D.; Dreizler, A. Flow field studies of a new series of turbulent premixed stratified flames. Combust. Flame 2010, 157, 384-396. [CrossRef]

10. Xue, G.H.; Chai, J.X. Temperature control error research based on thermocouple sensor. China Meas. Test. Technol. 2019, 45, $100-104$.

11. Jia, R.; Xiong, Q.; Xu, G.; Wang, K.; Liang, S. A method for two-dimensional temperature field distribution reconstruction. Appl. Therm. Eng. 2017, 111, 961-967. [CrossRef]

12. Bramanti, M.; Salerno, E.A.; Tonazzini, A.; Pasini, S.; Gray, A. An acoustic pyrometer system for tomographic thermal imaging in power plant boilers. IEEE Trans. Instrum. Meas. 1996, 45, 159-167. [CrossRef]

13. Ma, L.; Ning, H.; Wu, J.; Ren, W. In situ flame temperature measurements using a mid-infrared two-line $\mathrm{H}_{2} \mathrm{O}$ laser-absorption thermometry. Combust. Sci. Technol. 2017, 10, 17. [CrossRef]

14. Hossain, M.M.; Lu, G.; Yan, Y. Optical fiber imaging based tomographic reconstruction of burner flames. IEEE Trans. Instrum. Meas. 2012, 61, 1417-1425. [CrossRef]

15. Staff, C.C. Non-contact temperature measurement. J. Bacteriol. 2001, 183, 2025-2031.

16. Fuqiang, W.; Ziming, C.; Jianyu, T.; Yuan, Y.; Yong, S.; Linhua, L. Progress in concentrated solar power technology with parabolic trough collector system: A comprehensive review. Renew. Sustain. Energy Rev. 2017, 11, 1314-1328. [CrossRef]

17. Aharon, M.; Elad, M.; Bruckstein, A. K-SVD: An Algorithm for Designing Overcomplete Dictionaries for Sparse Representation. IEEE Trans. Signal Process. 2006, 54, 4311-4322. [CrossRef]

18. Berkooz, G.; Holmes, P.; Lumley, J.L. The Proper Orthogonal Decomposition in the Analysis of Turbulent Flows. Annu. Rev. Fluid Mech. 1993, 25, 539-575. [CrossRef]

19. Luh, G.C.; Lin, C.Y. PCA based immune networks for human face recognition. Appl. Soft Comput. 2011, 11, 1743-1752. [CrossRef]

20. Polansky, J.; Wang, M. Proper Orthogonal Decomposition as a technique for identifying two-phase flow pattern based on electrical impedance tomography. Flow Meas. Instrum. 2017, 53, 126-132. [CrossRef]

21. Sun, X.H.; Xu, M.H. Optimal control of water flooding reservoir using proper orthogonal decomposition. J. Comput. Appl. Math. 2017, 320, 120-137. [CrossRef] 
22. Chen, M.X.; Liu, S.; Sun, S.X.; Liu, Z.; Zhao, Y. Rapid Reconstruction of Simulated and Experimental Temperature Fields Based on Proper Orthogonal Decomposition. Appl. Sci. 2020, 10, 3729. [CrossRef]

23. Sun, S.; Liu, S.; Chen, M.; Guo, H. An Optimized Sensing Arrangement in Wind Field Reconstruction Using CFD and POD. IEEE Trans. Sustain. Energy 2020, 11, 2449-2456. [CrossRef]

24. Tucker, L.R. Implications of factor analysis of three-way matrices for measurement of change. Probl. Meas. Chang. 1963, 15, 122-137.

25. Maldonado-Chan, M.; Mendez-Vazquez, A.; Guardado-Medina, R.O. Multimodal tucker decomposition for gated rbm inference. Appl. Sci. 2021, 11, 7397. [CrossRef]

26. Li, R.; Pan, Z.; Wang, Y.; Wang, P. The correlation-based tucker decomposition for hyperspectral image compression. Neurocomputing 2021, 419, 357-370. [CrossRef]

27. Marmoret, A.; Cohen, J.E.; Bertin, N.; Bimbot, F. Uncovering audio patterns in music with Nonnegative Tucker Decomposition for structural segmentation. arXiv 2021, arXiv:2104.08580.

28. Zhao, M.; Li, W.; Li, L.; Tao, R. Infrared Small-Target Detection Based on Three-Order Tensor Creation and Tucker Decomposition. In Proceedings of the 2021 IEEE International Geoscience and Remote Sensing Symposium IGARSS, Brussels, Belgium, 11-16 July 2021; pp. 3129-3132.

29. Qin, L.; Liu, S.; Kang, Y.; Yan, S.A.; Schlaberg, H.I.; Wang, Z. Wind velocity distribution reconstruction using CFD database with Tucker decomposition and sensor measurement. Energy 2019, 167, 1236-1250. [CrossRef]

30. Hou, Z.; Li, W.; Tao, R.; Du, Q. Three-Order Tucker Decomposition and Reconstruction Detector for Unsupervised Hyperspectral Change Detection. IEEE J. Sel. Top. Appl. Earth Obs. Remote Sens. 2021, 14, 6194-6205. [CrossRef]

31. Zhang, X. Matrix Analysis and Application; Tsinghua University Press: Beijing, China, 2013; pp. $453-471$.

32. Workshop, T. Piloted CH4/Air Flames C, D, E and F. Available online: https://tnfworkshop.org/data-archives/pilotedjet/ch4 -air/ (accessed on 16 June 2021).

33. Barlow, R.S.; Meares, S.; Magnotti, G.; Cutcher, H.; Masri, A.R. Local extinction and near-field structure in piloted turbulent $\mathrm{CH} 4$ /air jet flames with inhomogeneous inlets. Combust. Flame 2015, 162, 3516-3540. [CrossRef] 